\title{
EL APRENDIZAJE DE LA CIUDADANÍA A LO LARGO DE LA VIDA: UN ESTUDIO NARRATIVO DESDE HISTORIAS DE VIDA
}

LIFELONG CITIZENSHIP LEARNING: A NARRATIVE STUDY OF LIVES

\author{
A APRENDIZAGEM DA CIDADANIA AO LONGO DA VIDA: \\ UM ESTUDO NARRATIVO A PARTIR DE HISTÓRIAS DE VIDA
}

\author{
Ana ARRAIZ PÉREZ*, Fernando SABIRÓN SIERRA* \& Verónica AZPILLAGA LARREA \\ *Universidad de Zaragoza, ${ }^{* *}$ Universidad del Pais Vasco
}

PALABRAS CLAVE: educación cívica narrativas personales investigación cualitativa aprendizaje a lo largo de la vida educación no-formal historia de vida

KEYWORDS:

civic education personal narratives qualitative research lifelong learning nonformal education life history

\footnotetext{
Fecha de recepción del artículo: 15.V.2014

Fecha de revisión del artículo: 2.v1.2014 Fecha de aceptación final: 8.iv.2015
}

RESUMEN: Esta investigación responde a dos objetivos: comprender la construcción de la identidad ciudadana, y extraer claves interpretativas de su aprendizaje como ciudadanos activos. El estudio se realiza a través de historias de vida, con un diseño de caso múltiple. Se utilizan tres entrevistas de orientación biográfica para elaborar cada una de las historias. El tratamiento cualitativo de los datos obedece a un doble análisis: un análisis secuencial, como reflejo de los procesos de construcción identitarios en la trayectoria vital; y otro categorial, como articulación del qué y del cómo aprenden su condición ciudadana. Los resultados del análisis secuencial arrojan trayectorias vitales que, en su singularidad, reflejan la identificación y preocupación constante por mejorar, de forma eficiente, el entorno próximo en el que se desenvuelven, así como la práctica continuada de acción social. Del análisis categorial emerge un modelo interpretativo distribuido en dos dimensiones interrelacionadas. De una parte, la dimensión "resultados de aprendizaje" con tres macrocategorías: la "acción", definida por el dominio de destrezas (liderazgo y poder, empatía y diálogo, iniciativa e innovación); el "compromiso", articulado desde determinados valores (vitalismo y viabilidad, sensibilidad y responsabilidad, regeneración y transformación); y la "identidad" (situada, discursiva y dialéctica). De otra parte, la dimensión "procesos de aprendizaje" con las siguientes macrocategorías: los "escenarios"; las "vivencias" y la "socialización. La discusión de resultados enfatiza los principios de la diversificación, del descontrol y de la autoría como referentes de una acción socio-cultural que estimule la formación ciudadana: Se trata de comprometer a los protagonistas para "hacer cosas" útiles, en escenarios próximos que ofrezcan la posibilidad de mejora; de promover acciones que propicien la auto-reflexión; y de concienciar sobre la complejidad de la propia realidad. Principios a aplicar en las experiencias educativas a lo largo de la trayectoria vital de las personas desde sus inicios.

ABSTRACT: The study has two objectives: to understand the construction of civic identity and draw some key conclusions for the interpretation of its learning as active citizens. The study was carried out through life stories, using a multiple-case design. Three biographical oriented interviews were used to prepare each of the stories. The qualitative handling of the data used dual analysis: sequential analysis, as a reflection of the identity construction processes throughout life, and categorical analysis as a connection between the "what" and the "how" people learn their civic identity. The results of the sequential analysis show life 


\begin{tabular}{|c|c|}
\hline & $\begin{array}{l}\text { spans that, in their singularity, reflect the constant identification and desire to improve, ef- } \\
\text { ficiently, the immediate surroundings where people develop, as well as the continuous prac- } \\
\text { tice of social action. The categorical analysis produced an interpretative model split into } \\
\text { two interrelated dimensions. On one hand the "learning results" dimension with three ma- } \\
\text { cro-categories: "action", defined by the possession of skills (leadership and power, empathy } \\
\text { and dialogue, initiative and innovation); "commitment", defined by certain values (vitality } \\
\text { and viability, sensitivity and responsibility, regeneration and transformation); and "identity" } \\
\text { (situated, discursive and dialectic). On the other hand, the "learning processes" dimension, } \\
\text { with the following macro-categories: "scenarios"; "life experiences" and "socialisation. The } \\
\text { discussion of results emphasises the principles of diversification, lack of control and aut- } \\
\text { horship as references for a socio-cultural action that will stimulate citizenship training. This } \\
\text { is based on making the protagonists "do useful things" in local scenarios that offer the op- } \\
\text { portunity for improvement, on promoting actions that will encourage self-reflection, and } \\
\text { on raising awareness about the complexity of reality itself. These are principles that can be } \\
\text { applied in educational experiences throughout people's life spans from their beginnings. }\end{array}$ \\
\hline $\begin{array}{l}\text { PALAVRAS-CHAVE: } \\
\text { educação comunitária } \\
\text { Narrativas pessoais } \\
\text { Investigação qualitativa } \\
\text { Aprendizagem ao } \\
\text { longo da vida } \\
\text { Educação não formal } \\
\text { Histórias de vida }\end{array}$ & $\begin{array}{l}\text { RESUMO: A investigação responde a dois objetivos: compreender a construção da identi- } \\
\text { dade cidadã e extrair pontos interpretativos da sua aprendizagem como cidadãos ativos.O } \\
\text { estudo é realizado através de histórias de vida, com um desenho de caso múltiple. Utili- } \\
\text { zam-se três entrevistas de orientação biográfica para elaborar cada uma das histórias. O tra- } \\
\text { tamento qualitativo dos dados obedece a uma dupla análise: uma análise sequencial, como } \\
\text { reflexo dos processos de construção identitários na trajetória vital, e a outra categoria, } \\
\text { como articulação do que e do como aprendem a sua condição cidadã. Os resultados da } \\
\text { análise sequencial lançam trajetórias vitais que, na sua singularidade, refletem a identifi- } \\
\text { cação e preocupação constante por melhorar, de forma eficiente, o contexto próximo onde } \\
\text { se desenvolvem, assim como a prática contínua da ação social. Da análise categorial emerge } \\
\text { um modelo interpretativo distribuído em duas dimensões inter-relacionadas. Por um lado, } \\
\text { a dimensão "resultados de aprendizagem" com três macrocategorias: a "ação", definida pelo } \\
\text { domínio de destrezas (liderança e poder, empatia e diálogo, iniciativa e inovação); o "com- } \\
\text { promisso", articulado a partir de determinados valores (vitalismo e viabilidade, sensibili- } \\
\text { dade e responsabilidade, regeneração e transformação); e a "identidade" (situada, discursiva } \\
\text { e dialética). Por outro lado, a dimensão "processos de aprendizagem" com as seguintes ma- } \\
\text { crocategorias: os "cenários", as "vivências"; e a "socialização. A discussão dos resultados } \\
\text { enfatiza os princípios da diversificação, do descontrolo e da autoria como referentes de } \\
\text { uma ação sociocultural que estimule a formação cidadã: trata-se de comprometer os pro- } \\
\text { tagonistas para "fazerem coisas" úteis, em cenários próximos que ofereçam a possibilidade } \\
\text { de melhoria; de promover açães que propiciem a autorreflexão e de consciencializar acerca } \\
\text { da complexidade da própria realidade. Princípios a aplicar nas experiências educativas ao } \\
\text { longo da trajetória vital das pessoas desde o seu início. }\end{array}$ \\
\hline
\end{tabular}

\section{Introducción}

La investigación, en la que se insertan las historias de vida, se desarrolla en torno al aprendizaje de la ciudadanía activa; perspectiva que, superando la clásica concepción individualista de ciudadanía, promueve la educación como práctica ciudadana. Línea que pretende superar limitaciones y problemas de planteamientos previos sobre la educación de la ciudadanía (Lawy \& Biesta, 2006). La finalidad del proyecto es la formulación de estrategias eficaces para el aprendizaje de la ciudadanía activa mediante la identificación y el análisis de las prácticas y experiencias a través de las cuales se aprende a ser y actuar como ciudadano/a'. El trabajo de campo se articula en torno a cuatro cuestiones de investigación: "1) qué es, qué significa ser ciudadana o ciudadano; 2) cómo se ejerce la ciudadanía y qué actividades implica; 3) en qué principios, valores y motivaciones se basa su ejercicio; y 4) de qué formas se aprende y qué recursos, medios, habilidades se ponen en juego" (Mata \& Gil-Jaurena, en prensa). Las historias de vida indagan sobre esta última cuestión referida al aprendizaje, a la vez que permiten contrastar los resultados relativos a las otras tres cuestiones de investigación. De manera específica, el estudio tiene un doble objetivo: comprender las vivencias, los significados en la construcción de la identidad ciudadana; y extraer claves interpretativas de su aprendizaje como ciudadanos activos y participativos.

La opción por las historias de vida obedece a dos sentidos intrínsecos al proyecto de investigación: una razón metodológica y un interés formativo. En el plano metodológico, las historias de vida son pertinentes para el establecimiento de las categorías que, desde su condición de ciudadanos considerados ejemplares, puedan dar cuenta del aprendizaje de la ciudadanía. Esta misma ejemplaridad -en el sentido "cotidiano" de ejemplar (Ferrarotti, 2009)- tiene un carácter ilustrativo de alto potencial de las historias de vida en la educación formal, no-formal e informal (Josso, 2002). Esta doble utilidad, tanto investigadora como formativa, caracteriza a las historias de vida desde su origen antropológico (Morin, 1980), a lo largo de su desarrollo con el interaccionismo simbólico (Becker, 1974), y se mantiene en la impronta actual del método narrativo en la orientación para la construcción personal de la realidad (Peavy, 1998). 
El aprendizaje de la ciudadanía es dinámico e inacabado, se realiza a lo largo de la vida de la persona; a la vez que poliédrico, pues confluyen dimensiones sistémicas, coyunturales y personales que fraguan un particular aprendizaje a lo largo de la trayectoria vital de la persona. El relato construido desde las historias de vida expresa ese recorrido existencial en la intersección entre la investigación y la formación, el aprendizaje y la acción (Pineau, 2006). Sobre estos enunciados teórico-epistemológicos, se están desarrollando proyectos colectivos de investigación en los que se indaga-específicamente- sobre el aprendizaje de la ciudadanía desde las historias de vida ${ }^{2}$. En nuestro caso, el estudio que se presenta se adscribe a esta línea emergente de investigación, reforzando resultados de estudios previos análogos (Holford \& Veen, 2003), a la vez que se profundiza en la multirreferencialidad del tema y en la voz crítica de los informantes.

\section{El método narrativo}

\subsection{Diseño}

El estudio se desarrolla desde un enfoque narrativo en el que las historias de vida articulan un diseño de estudio de caso múltiple (Stake, 1998). Cada una de las tres historias de vida presenta un caso (intra) que, a su vez, se satura y contrasta con las dos restantes (inter) (Huberman \& Miles, 1991). El énfasis metodológico del estudio hace referencia a la "función analítica" de las historias de vida como una de las claves de la "teoría fundamentada en los hechos" (Bertaux, 1989). El principio de la "teoría fundamentada en los hechos" (Glaser \& Strauss, 2002) por el que una teoría puede "emerger" de la realidad (frente a la teoría que se aplica a la explicación de la realidad), es pertinente por cuanto permite indagar sobre el cómo y el por qué determinados ciudadanos considerados ejemplares aprenden, son y actúan como lo hacen.

\subsection{Informantes}

La cuestión decisiva en torno a la credibilidad científica de las historias de vida no está tanto en el número de historias de vida, sino más bien en la manera en la que se han realizado (Peneff, 1990; Bertaux, 1999; Villers, 2011). No se trata de buscar la representatividad por el número de informantes (muestreo estadístico), sino por la relevancia del informante en el tema de investigación (muestreo teórico). En este caso, tras realizar una primera fase de trabajo de campo con entrevistas en profundidad, el criterio de selección básico de los informantes fue su condición de ejemplares en su participación, tanto para el grupo de investigación como para los entrevistados en la primera fase del trabajo, con indicadores claros de su identificación y vinculación directa con proyectos de in- tervención y mejora comunitaria. En el estudio de caso múltiple han participado tres informantes claves (María, Marta y Miguel) 3 .

Los informantes tienen, en sus perfiles, matices diferenciales que, entre otros, se manifiestan en los escenarios físicos en los que se desenvuelve su vida (medio rural-urbano; universidad-empresa privada), en la edad (de 35 a 60 años), mayor o menor estabilidad laboral, formación en diferentes ámbitos profesionales, y diferentes responsabilidades familiares. Son dos mujeres y un hombre.

\subsection{Proceso de elaboración de las historias de vida}

La elaboración de sus historias de vida se ha realizado a partir de entrevistas de orientación biográfica en tres momentos clave: Una primera tanda de entrevistas abiertas en las que la persona cuenta su vida con el estímulo del tema de la ciudadanía, y el investigador escucha. Tras la audición y un primer análisis intuitivo, una segunda tanda, más conversacional, en la que se indaga con mayor focalización en el tema de la ciudadanía a lo largo de su trayectoria vital, su vinculación con las dimensiones personales, los contextos y las acciones en los que se desarrolla su vida. En un tercer momento, el texto elaborado por el grupo de investigación sobre las transcripciones de las entrevistas, se contrasta con el informante. Este último paso es determinante en la validez y credibilidad de las historias de vida: El proceso de análisis se realiza sobre el texto construido conjuntamente por investigadores e investigados; es decir, sobre la narración socioconstruida por las personas que han intervenido.

\subsection{Proceso de análisis de las historias de vida}

El análisis se desarrolla desde una perspectiva interpretativa (Marie-Claude, 2014) y un tratamiento cualitativo de las historias de vida bajo dos ejes, diacrónico y sincrónico (Villers, 2011): El primero, secuencial, da cuenta de los avatares existenciales de la persona que narra la historia de su vida; el segundo, en el que se organiza, define y relaciona el sistema categorial emergente de las tres historias de vida. El eje secuencial documenta la construcción de la identidad de cada persona y explicita los procesos singulares de aprendizaje; el sistema de categorías refiere procesos y productos comunes al aprendizaje de nuestros informantes. Esta aproximación inter-intra caso (Huberman \& Miles, 1991) refuerza la validez intersubjetiva 4 de las categorías emergentes. El proceso de categorización se ha realizado siguiendo el "método comparativo constante" aplicado a la transcripción y codificación de cada uno de los textos de las respectivas historias de vida, utilizando para ello el software NVivo (Richards, 2011). El proceso de codificación se 
realiza por dos miembros del equipo de investigación que, de manera separada, iban construyendo primero los temas, después las precategorías, las categorías iniciales y las categorías finales resultantes. La relación intercategorial se establece triangulando el contenido de las categorías finales con cada uno de los relatos de las historias de vida. El proceso de validación concluye con un contraste tanto con los informantes, como con los miembros del equipo de investigación que habían realizado las historias de vida.

\section{Resultados: el aprendizaje de la ciuda- danía emergente de tres historias de vida}

Los resultados se presentan en dos propuestas: Desde el análisis secuencial, como reflejo de los procesos de construcción identitarios en la trayectoria vital de nuestros informantes; se responde así al objetivo indagatorio. Desde el análisis categorial, como articulación del qué y del cómo aprenden y desarrollan los informantes su condición de ciudadanos activos y participativos; se responde aquí al objetivo de extraer claves interpretativas.

\subsection{El análisis secuencial de las historias de vida: la identidad ciudadana}

Marta es la primera informante. De sus relatos se desprende una construcción de la identidad que evoluciona, con un movimiento cíclico y dialéctico, de dentro-a-fuera y de fuera-a-dentro, en una búsqueda permanente de sentirse más a gusto. La reflexión sobre las realidades, contrastada con lecturas, informaciones y observaciones, supone el estímulo para la acción; acción en red desde la participación horizontal como estrategia para promover el cambio de la realidad. La vivencia de las acciones le cuestionan las limitaciones de las redes y le abren posibilidades para evolucionar y crecer con otras redes: $A$ ver con quién me puedo aliar para hacer esto... El motor es la comunicación con los otros, la crítica y auto-crítica: Estuve haciendo alguna cosa de éstas, de visitar a gente mayor, ... imagino yo también que esto han sido cosas que he estado procesando. Dejé después ese grupo. Llega un momento en el que has cogido lo que necesitabas. El sentido de la acción es mejorar el entorno, en una espiral que arranca del entorno próximo, se abre a un entorno más amplio, para ir cerrándose de nuevo hacia lo más próximo: Yo tenía que hacer algo para cambiar las cosas. Se inicia con la organización doméstica en la familia, continúa en organizaciones sociales, la militancia política de proyección internacional para retornar a las asociaciones vecinales del barrio. Hay igualmente una constante de proximidad-distanciamiento a los otros, entrecruzando ideas, y afectos: Me dí cuenta de que los sentimientos son algo muy importante; $y$ es algo que no entendía en mis primeras etapas participativas. Ha- cía una práctica des de la cabeza, y me dícuenta de que tenía que complementar y hacerlo desde el corazón y el estómago. Los hitos en la vida de Marta le deben mucho a la dimensión emocional con los demás. Actúa y evoluciona en contacto con los demás. En su vida, le confiere mucha importancia a los libros. Son las ideas y la comunicación con los demás: Yo intento debatir, debatir y proponer. La participación horizontal es el referente clave de su evolución. En la actualidad, se presenta desencantada de la vida política. Evoluciona de instituciones formales a menos formales: Eso de que alguien por arriba decidiera algo y yo, por narices tuviera que hacerlo, pues no.

María es la segunda informante. Ofrece una construcción de la identidad desde el conflicto y el cambio como búsqueda de realización personal: Tuve otra época muy personal de búsqueda interna hacia donde quería ir, porque entré en conflicto con mi profesión, entré en conflicto con mi modo de actuar ante la vida y quería buscar un lugar en el que yo me sintiera realizada. El afecto de los otros, las experiencias profesionales y formativas alimentan su cuestionamiento personal centrado en el qué hago, cómo lo estoy haciendo o cómo lo puedo hacer y dónde lo estoy haciendo. De esa dialéctica entre las experiencias con los demás y su cuestionamiento surgen las crisis que suponen un momento de renovación personal y de crecimiento: El mundo se cambia desde tu espacio, en esa zona de desarrollo próximo es donde yo creo que hay que actuar. Las crisis pueden orientarse hacia momentos o etapas en las que prima la dependencia emocional de la informante, e incluso económica; y en otras va a primar la independencia y el yo; ambas tienen, sin embargo, el mismo sentido, el entrelazado entre su propio desarrollo emocional con su misión de hacer cosas en su doble compromiso consigo misma y con el entorno próximo: Cambié lo que estuvo en mi mano y desaparecí justo en el momento en el que creí que había hecho todo lo que estaba en mi mano. El gran motor en la construcción de su identidad es la automayéutica, la interrogación consigo misma y con los demás: Trato siempre de crear la pregunta. Porque uno no es consciente de cómo es, de cómo actúa en la vida hasta que no hay una persona que le dice ¿por qué acabas de actuar así? La vida de María son constantes rupturas sentimentales y rupturas profesionales cuando se pregunta los porqués de las experiencias vividas; necesita viajar, conocer, sentir para ampliar los sentidos de su interrogación: Uno tiene que sentirse protagonista activo de su vida para realmente cambiar algo. Y necesita llenar su saco de experiencias, de sentimientos y de la cultura y la formación. María va evolucionando, se cuestiona, se realiza al actuar con los demás y para los demás, en una espiral de cambio que avanza hacia el futuro: Entonces creo que ahí es donde puedo aportar, en el cuestionamiento, en la reflexión y en la posibilidad de construir con otros. 
Miguel es el tercer informante. Su biografía relata, al igual que la de Marta y María, una construcción dialéctica de la identidad, bajo el movimiento constante entre la proyección y la apertura hacia el exterior con el contrapunto del repliegue hacia sí mismo, en momentos cotidianos de soledad incluso física. Distribuye sus tiempos (incluso su horario) entre los paseos en solitario y las actividades con los demás. Destaca en Miguel la acción comprometida con su entorno próximo, que llega a un fuerte compromiso con el territorio. En este compromiso hay también una búsqueda por encontrarse bien: Yo lo he pasado mal cuando otros lo han pasado mal, pero a la vez tenía que estar yo bien. $Y$ si estoy yo bien, yo sé que la gente a mi alrededor también lo está; me ha tocado un poco un papel siempre de cierto liderazgo. Es una vinculación afectiva, que arranca en su familia y mantiene con sus parejas (sic) e hijos. Destacan en su construcción cíclica de la identidad, dos constantes: la consideración de las limitaciones de la realidad que le toca vivir como estímulo para las acciones; y la planificación de futuro como un eje esencial de su vida. Necesito tener [algo] cada año en la cabeza para tener esas ilusiones para poder [vivir]. Para mí ha sido fundamental siempre tener objetivos; objetivos vivenciales [que] para mí eran mis proyectos. La identidad de Miguel está marcada con la idea de proyecto; que le permite conjugar el compromiso territorial, social y personal, con su visión de oportunidad en la percepción de las realidades: Siempre he querido anticiparme a los acontecimientos. Hay un ingrediente de reflexión sobre su propia acción y del sentido que tiene la acción en el entorno personal y próximo. Esta reflexión es motor de cambio en las personas con las que se involucra y en las asociaciones que crea. Su identidad se desarrolla desde las vivencias en escenarios geográficos muy diferenciados, que delimitan con más fuerza el territorio de pertenencia (ej.: la ruralidad adquiere sentido cuando se traslada a la ciudad). Viaja mucho. Está alerta a lo que ocurre en el mundo. Entiende la comunicación como instrumento de relación y de aprendizaje. Miguel siempre aprende de los demás. Se rebela contra lo que entiende que es una injusticia; a la vez, cree incondicionalmente en las personas: $y$ trabajé con mucha de esa gente ruda que había conocido de crío y les tenía como mucho mucho respeto, por un lado y, por otro lado, me daban cierta ternura, pena. Es una persona muy reconciliada con su propia historia; y cuanto más se siente en armonía, más se proyecta en el futuro. Esencialmente, es un vitalista: Vive, a la vez, deja vivir y procura que te dejen vivir.

La trayectoria vital de Marta, María y Miguel tiene, en su singularidad, unos hitos vitales que se recogen en lenguaje telegráfico (figura 1). La descripción refleja la identificación y preocupación constante por mejorar, de forma eficiente, el entorno próximo en el que se desenvuelven, así como la práctica continuada de acción social en todas las etapas de su vida.

\begin{tabular}{|c|c|c|c|c|}
\hline \multicolumn{5}{|c|}{ Figura 1: La trayectoria vital de Marta, María y Miguel } \\
\hline & INFANCIA & ADOLESCENCIA & JUVENTUD & MADUREZ \\
\hline \multirow{3}{*}{ Nicho ecológico } & $\begin{array}{l}\text { (MARTA) } \\
\text { Nace en ciudad. Am- } \\
\text { biente familiar afectivo y } \\
\text { comprometido.La es- } \\
\text { cuela es acogedora. }\end{array}$ & $\begin{array}{l}\text { Realza instituto y } \\
\text { relación con } \\
\text { los compañeros. }\end{array}$ & $\begin{array}{l}\text { Compagina vida en } \\
\text { pareja y voluntariado. }\end{array}$ & $\begin{array}{l}\text { Estancia país extranjero- } \\
\text { retorno ciudad de ori- } \\
\text { gen-estancia en un } \\
\text { nuevo país-retorno. } \\
\text { Vuelta a la universidad. } \\
\text { Destaca familia, amigos y } \\
\text { nuevos amigos. }\end{array}$ \\
\hline & $\begin{array}{l}\text { (MARÍA) } \\
\text { Nace en otro país. Am- } \\
\text { biente familiar intelec- } \\
\text { tual (muy lectores). }\end{array}$ & $\begin{array}{l}\text { Aislamiento en contexto } \\
\text { familiar. Destaca insti- } \\
\text { tuto y pandilla. }\end{array}$ & $\begin{array}{l}\text { Se traslada de país por } \\
\text { trabajo. Participa en or- } \\
\text { ganizaciones estudianti- } \\
\text { les. Milita en partido po- } \\
\text { lítico. }\end{array}$ & $\begin{array}{l}\text { Familia propia. Asocia- } \\
\text { ciones de padres. } \\
\text { Asociaciones vecinales. }\end{array}$ \\
\hline & $\begin{array}{l}\text { (MIGUEL) } \\
\text { Nace en pueblo de mon- } \\
\text { taña. Familia numerosa. }\end{array}$ & $\begin{array}{l}\text { Traslado familiar forzoso } \\
\text { a la ciudad. Scouts. Se } \\
\text { acerca a organizaciones } \\
\text { estudiantiles. }\end{array}$ & $\begin{array}{l}\text { Implicación en estructu- } \\
\text { ras universitarias (claus- } \\
\text { trable). Retornos cons- } \\
\text { tantes al pueblo. }\end{array}$ & $\begin{array}{l}\text { Vuelta a los escenarios } \\
\text { de la infancia. Crea su } \\
\text { propia familia. Consolida } \\
\text { el trabajo en coopera- } \\
\text { tiva. Se incorpora al } \\
\text { ayuntamiento y a organi- } \\
\text { zaciones sociales diver- } \\
\text { sas. Entiende la montaña } \\
\text { como escenario de pro- } \\
\text { yección y reflexión. }\end{array}$ \\
\hline
\end{tabular}




\begin{tabular}{|c|c|c|c|c|}
\hline \multirow{3}{*}{ Vivencias } & $\begin{array}{l}\text { (MARTA) } \\
\text { Sentirse querida. Adap- } \\
\text { tación escolar. }\end{array}$ & $\begin{array}{l}\text { Desadaptación escolar. } \\
\text { Soledad. Querer hacer } \\
\text { grandes cosas y no sa- } \\
\text { ber ni poder. }\end{array}$ & $\begin{array}{l}\text { Independencia familiar- } \\
\text { dependencia de pareja. } \\
\text { Autonomía económico- } \\
\text { laboral. Ruptura y cam- } \\
\text { bio de escenarios. Aper- } \\
\text { tura a los otros y al terri- } \\
\text { torio-crisis aislamiento. }\end{array}$ & $\begin{array}{l}\text { Dinamización universita- } \\
\text { ria. La formación como } \\
\text { necesidad para hacer. } \\
\text { Importancia de sentirse } \\
\text { autónoma. }\end{array}$ \\
\hline & $\begin{array}{l}\text { (MARÍA) } \\
\text { Observar y reflexionar } \\
\text { situaciones cotidianas. } \\
\text { Aislamiento del entorno, } \\
\text { introversión. }\end{array}$ & $\begin{array}{l}\text { Responsabilidad existen- } \\
\text { cial ante las situaciones } \\
\text { de injusticias. Ubicación } \\
\text { en la pandilla para ha- } \\
\text { blar. }\end{array}$ & $\begin{array}{l}\text { Alfabetización política de } \\
\text { partidos y organizacio- } \\
\text { nes. Compromiso senti- } \\
\text { mental vinculado a su } \\
\text { compromiso social. De- } \\
\text { sarrollo responsable en } \\
\text { compromiso social. Im- } \\
\text { portancia de las lecturas. }\end{array}$ & $\begin{array}{l}\text { Desencanto partidos po- } \\
\text { líticos. Participación de- } \\
\text { mocrática-horizontal. }\end{array}$ \\
\hline & $\begin{array}{l}\text { (MIGUEL) } \\
\text { Adaptación al medio na- } \\
\text { tural. Maravillarse por las } \\
\text { cosas y las personas. Co- } \\
\text { nocer personas y situa- } \\
\text { ciones diversas. }\end{array}$ & $\begin{array}{l}\text { Desubicación. Inquietud } \\
\text { por conocer y hacer. } \\
\text { Consecuencias de la po- } \\
\text { lítica en la vida coti- } \\
\text { diana.El poder del dis- } \\
\text { curso. }\end{array}$ & $\begin{array}{l}\text { Activismo político-estu- } \\
\text { diantil y liderazgo. Com- } \\
\text { promiso con las necesi- } \\
\text { dades familiares.Desin- } \\
\text { terés curricular vs. } \\
\text { aprender de las perso- } \\
\text { nas.Contradicciones } \\
\text { asumidas. }\end{array}$ & $\begin{array}{l}\text { Responsabilidad familiar } \\
\text { incrementada. Respon- } \\
\text { sabilidades laborales } \\
\text { como empresario. Asu- } \\
\text { mir poder para hacer, } \\
\text { nunca para estancarse. } \\
\text { Saber quién es, por qué } \\
\text { está y cuándo debe irse. }\end{array}$ \\
\hline \multirow{3}{*}{ Claves } & $\begin{array}{l}\text { (MARTA) } \\
\text { Modelos familiares: res- } \\
\text { ponsabilidad con la reali- } \\
\text { dad. La afectividad } \\
\text { como motor. }\end{array}$ & $\begin{array}{l}\text { Incomprensión, soledad, } \\
\text { distanciamiento. Bús- } \\
\text { queda de la autonomía e } \\
\text { independencia. }\end{array}$ & $\begin{array}{l}\text { Compromiso con lo coti- } \\
\text { diano. Apertura a expe- } \\
\text { riencias, gentes y am- } \\
\text { bientes diversos. Crisis, } \\
\text { rupturas y proyectos. } \\
\text { Aprende desde la acción } \\
\text { cuestionada. }\end{array}$ & $\begin{array}{l}\text { Asumir la historia, refle- } \\
\text { xionar, cuestionar, hacer, } \\
\text { formarse. }\end{array}$ \\
\hline & $\begin{array}{l}\text { (MARÍA) } \\
\text { Lecturas: los héroes } \\
\text { como modelos de com- } \\
\text { portamiento. }\end{array}$ & $\begin{array}{l}\text { Concienciación de las in- } \\
\text { justicias mundiales y do- } \\
\text { mésticas desde el aisla- } \\
\text { miento.El debate como } \\
\text { herramienta de acción } \\
\text { con los otros. }\end{array}$ & $\begin{array}{l}\text { Activismo participativo } \\
\text { desde: } 10 \text { Observar para } \\
\text { aprender, } 20 \text { Liderazgo } \\
\text { como iniciativa y estímulo } \\
\text { para los otros, } 30 \text { Reti- } \\
\text { rada y ruptura como con- } \\
\text { secuencia de las contra- } \\
\text { dicciones organizativas. }\end{array}$ & $\begin{array}{l}\text { Vuelta a lo próximo (al } \\
\text { colegio y al barrio).Bús- } \\
\text { queda de la participa- } \\
\text { ción horizontal (hacer } \\
\text { con). Énfasis en la crítica } \\
\text { y los retos. Integración } \\
\text { cognición y emoción en } \\
\text { la acción. }\end{array}$ \\
\hline & $\begin{array}{l}\text { (MIGUEL) } \\
\text { Responsabilidad en las } \\
\text { necesidades familiares. }\end{array}$ & $\begin{array}{l}\text { Añoranza del territorio y } \\
\text { rebeldía. Lecturas para } \\
\text { fundamentar su dis- } \\
\text { curso.Experiencias posi- } \\
\text { tivas de liderazgo. }\end{array}$ & $\begin{array}{l}\text { Responsabilidad con la } \\
\text { familia. Conocer y } \\
\text { aprender, argumen- } \\
\text { tando. Justicia social. } \\
\text { Coherencia y supera- } \\
\text { ción. Amistad. Empresa } \\
\text { familiar. }\end{array}$ & $\begin{array}{l}\text { Valor coyuntural de roles } \\
\text { y organizaciones por pro- } \\
\text { yectos. Sentido de las } \\
\text { personas. Apego al terri- } \\
\text { torio y obligación de via- } \\
\text { jar para conocer. Sus hi- } \\
\text { jos como clave de futuro. } \\
\text { Se anticipa y proyecta. }\end{array}$ \\
\hline
\end{tabular}




\subsection{El análisis categorial: el aprendizaje de la ciudadanía}

El modelo emergente se organiza en seis macro-categorías que, con el contenido que le atribuyen los informantes, se desglosan hasta un total de 17 categorías (figura 2).

Los resultados de aprendizaje implican la adquisición y desarrollo de una serie de componentes intrínsecos a los ciudadanos ejemplares. Se manifiestan en tres macro-categorías: acción, compromiso e identidad.

LA ACCIÓN. Representa la adquisición y el dominio de estrategias, habilidades y destrezas necesarias para articular aquellos comportamientos intencionales que suponen ejercer la ciudadanía activa con precisión. Incluye, a su vez, las siguientes categorías:

- Liderazgo y poder: Como estrategias para movilizar personas, recursos y medios necesarios para conseguir que se actúe. Las acciones siempre repercuten en el bien de la colectividad. Estos ciudadanos se autoproclaman líderes para ejercer una labor de mediación situada hacia el colectivo. Se corresponde con la "capacidad de agencia". Se trata de la posibilidad para movilizar a que otros participen, no un poder asociado a la imposición de alguien.

- Empatía y diálogo: Como habilidades comunicativas que permiten construir una realidad compartida entre el colectivo. Estos informantes, en su deseo de incorporar al otro, deben aproximarse a los demás (empatía). De esta competencia emanará la capacidad de liderazgo que le atribuyen los demás, porque se sienten comprendidos; a la vez que refleja la intencionalidad de hacer desde las necesidades explícitas o implícitas del grupo y que conviene primero desvelar. Los demás, se comprometen con ellos en procesos dialógicos de los que emerge la acción.

Figura 2: Análisis intra-categorial: las categorías emergentes

\begin{tabular}{|c|c|c|}
\hline \multicolumn{3}{|c|}{ Resultados } \\
\hline $\begin{array}{l}\text { ACCIÓN } \\
\text { - Liderazgo y poder } \\
\text { - Empatía y diálogo } \\
\text { - Iniciativa e innova- } \\
\text { ción }\end{array}$ & $\begin{array}{l}\text { COMPROMISO } \\
\text { - Vitalismo y vitalidad } \\
\text { - Sensibilidad y res- } \\
\text { ponsabilidad } \\
\text { - Regeneración y } \\
\text { transformación }\end{array}$ & $\begin{array}{l}\text { IDENTIDAD } \\
\text { - Situada } \\
\text { - Discursiva } \\
\text { - Dialéctica }\end{array}$ \\
\hline $\begin{array}{l}\text { ESCENARIOS } \\
\text { - Ecológicos } \\
\text { - Institución } \\
\text { - Comunidad }\end{array}$ & $\begin{array}{l}\text { VIVENCIAS } \\
\text { - Perfil experiencias } \\
\text { - Modos de autoría }\end{array}$ & $\begin{array}{l}\text { SOCIALIZACIÓN } \\
\text { - Alfabetización } \\
\text { - Interacción } \\
\text { - Cambio }\end{array}$ \\
\hline \multicolumn{3}{|c|}{ Procesos } \\
\hline
\end{tabular}

- Iniciativa e innovación: Como destrezas que aplicadas a la acción posibilitan avanzar al colectivo. Se refieren a la planificación continua de proyectos, con objetivos realistas, viables y novedosos. El ciudadano agente promueve cambios relacionales que favorecen nuevas dinámicas. En efecto, sus iniciativas son esencialmente de dinamización mediada en el colectivo, y sus procesos tienen ese carácter relacional innovador.

EL COMPROMISO. Se trata de los valores preferentes que subyacen en la motivación para la acción ciudadana; coexisten con otros valores dentro de su personal escala axiológica, pero son los esenciales en la implicación ciudadana. Esta macro-competencia se desglosa en un triple compromiso:

- Vitalismo y viabilidad: Es el valor de la Vida. La trayectoria vital se va sucediendo desde las posibilidades y resultados de la propia acción. El vitalismo, entendido como lo positivo, lo posible, el crecimiento y el cambio que dinamiza la existencia humana. El compromiso ciudadano es un compromiso personal para crecer y realizarse; siempre cabe la posibilidad de hacer algo en provecho de su vida y la de los demás.

- Sensibilidad y responsabilidad: Es el valor del Otro en su diversidad. El ciudadano activo y participativo es sensible hacia las condiciones del otro, asume la responsabilidad para que las personas, valiosas per se, no sufran las condiciones coyunturales que pueda imponer el sistema. Son sensibles no solo al colectivo sino que se muestran responsables frente a la particularidad de las personas que le rodean.

- Regeneración y transformación: Es el valor del Mundo. Recogen el bagaje histórico desde la familia, actúan en el presente colectivo con los demás, y bajo una perspectiva de mejorar el mundo para las siguientes generaciones. Hay un compromiso reforzado por los movimientos globalizadores-localistas, en términos tanto económicos como multiculturales en lo global y transgeneracionales en lo local.

LA IDENTIDAD. Está representada en la pregunta ¿quién soy yo?, a la que responden estos informantes: yo soy los papeles que represento en mi vida. Es una construcción en la interdependencia y la comunicación. El perfil de la identidad se define en las siguientes categorías:

- Situada. Como identidad contextualizada. Emerge del ejercicio simultáneo de roles en distintos escenarios. Se trata de la vinculación con el espacio social, con las instituciones, comunidades y grupos en 
que se desenvuelve; y se amplía al espacio físico. Aprenden a desempeñar múltiples roles interdependientes que les ofrecen posibilidades, necesidades y retos; tejiendo una red que constituye su identidad.

- Discursiva. Como identidad construida por el sentido pasado, presente y futuro de su existencia. Estos informantes elaboran una narración selectiva de experiencias, acompañadas de pensamientos y sentimientos que dan sentido a su propia vida. En sus discursos se percibe integración, coherencia e interpretación positiva. La trayectoria vital reflexionada se constituye en historia argumentada, que confiere singularidad y permanencia a su identidad.

- Dialéctica. Como identidad en proceso permanente de deconstrucción-reconstrucción a partir de las crisis, los conflictos, las contradicciones, y los dilemas que emergen cuando narran su trayectoria vital. Destacan las dinámicas dialécticas de apertura a los otros-distanciamiento, de acción-reflexión, de enseñar-aprender. Los ciudadanos activos y participativos son personas en permanente proceso de transformación.

De manera complementaria con los resultados, los procesos de aprendizaje integran las categorías que permiten explicar cómo aprenden los informantes el ejercicio de una ciudadanía. Tales procesos se dan bajo unas coordenadas espacio-temporales (los escenarios), comprometen un contenido que les da sentido (socialización), e implican la movilización de los propios procesos internos (las vivencias).

LOS ESCENARIOS. Constituyen el "nicho" de los procesos de aprendizaje. Son el medio físico, organizativo y comunitario donde se desarrollan interacciones y proyectos. La historia de vida de estos informantes se caracteriza por la amplitud de escenarios y su diversidad. La macro-categoría se desglosa en tres categorías emergentes:

- Escenarios ecológicos. Referidos a la relación de las personas con los ecosistemas. Los informantes entienden esa relación desde el equilibrio, la sostenibilidad y la regeneración. Hay una consideración positiva, sentida y necesaria para su bienestar personal, con una doble valencia: de una parte, la armonía con el ecosistema y las posibilidades de hacer cosas, de sentir y de pensar; de otra, el desequilibrio ecológico que impulsa proyectos centrados en retos a superar y necesidades a cubrir.

- Escenarios institucionales. Representada por las organizaciones que asignan un rol formal, convencional y legítimo a cada uno de sus miembros. El significado atribuido a estos roles es inicialmente asimilado, para ser repensado y reconstruido a la luz de otros referentes (incluso ecológicos), en un ejercicio de participación en la construcción de la realidad social. Los informantes coinciden en el potencial sobresaliente para el aprendizaje ciudadano de la familia y más limitado de la escuela e incluso de los partidos políticos. La plasticidad del rol, como margen de reconstrucción, condiciona la relevancia conferida por nuestros informantes.

- Escenarios comunitarios. Considerados los grupos y organizaciones con carácter voluntario de pertenencia, y planteamientos más abiertos en la definición de miembro que los escenarios anteriores. En algunos casos pretenden incorporar el debate constructivo como forma de funcionamiento. La creciente formalización (burocratización) de organizaciones a priori no-formales supone, para nuestros personajes, una contradicción. Pese a ello, estos escenarios ofrecen la posibilidad y viabilidad de acciones en pro de una participación horizontal que redunda en el cambio de los roles instituidos (participación vertical).

LAS VIVENCIAS. Constituyen la apropiación de las acciones que lleva a cabo el protagonista. El doble sentido de autoría se desarrolla tanto desde el perfil de las experiencias, cuanto por los modos de hacer y sentir la vivencia. Ambas categorías se delimitan a través de tres componentes:

- Perfil de las experiencias especificado en tres rasgos que las caracterizan:

- Diversificadas, por la variedad de personas o colectivos con quienes se relacionan y los distintos escenarios donde participan. El ciudadano va construyendo una red personal al conectarlas.

- Pragmáticas, porque resultan vivencias útiles para los colectivos y para ellos mismos. Cabe señalar que es el propio informante quien le atribuye y determina el sentido de la utilidad.

- Formativas, porque suponen la emergencia de dilemas y contradicciones sobre los que basan su propia reflexión y motivan los cambios que caracterizan sus trayectorias vitales.

- Modos de autoría organizados en tres situaciones:

- Por estar: El actor es esencialmente un observador-participante. Accede al escenario por proximidad y oportunidad; empieza a interaccionar y a conocer las posibilidades y necesidades que le ofrece. Predomina la reflexión sobre lo observado, desde la que construye proyectos.

- Por protagonizar: El actor experimenta. Aplica proyectos con la doble finalidad no sólo de hacer, sino de avanzar, de progresar (de ahí la ver- 
tiente de experimentación). Son acciones con matices innovadores.

- Por distanciarse: El autor se aísla y retorna a sí mismo. La evaluación y la reflexión que los informantes realizan de sí mismos se viven en términos positivos, como autorrefuerzo. Se valoran logros y aparentes fracasos, para iniciar acciones que les hagan avanzar en su trayectoria vital.

LA SOCIALIZACIÓN. Representa el tema de los procesos vivenciados. Es el proceso de enculturación en una sociedad ciudadana activa y participativa. Se incide en el carácter constructivista esbozado en el siguiente continuo representativo de las tres categorías obtenidas: del observo a los que están en una manifestación, acompaño a un compañero, y asumo cierto protagonismo al promover la incorporación de otros a la manifestación.

- Alfabetización, relativa a los procesos de aprendizaje instrumental, de aquellas herramientas que permitan el posterior desarrollo de la acción. El ciudadano adquiere lenguajes, normas, reglas, códigos, usos, ritos y rituales propios de una cultura activa y participativa.

- Interacción, definida como aquellas relaciones mantenidas desde la reciprocidad. Los intercambios se plantean en un plano de igualdad; se destaca el componente afectivo, con referencias sentimentales explícitas. La interacción recíproca se vincula a la horizontalidad como eje vertebrador de la ciudadanía activa.

- Cambio, define el significado de la socialización. El ciudadano interioriza el principio de la participación que tiene su sentido y razón de ser en el progreso de la sociedad. Se promueven acciones responsables para su propio futuro y el de las generaciones siguientes. Este referente de futuro da una proyección distinta a su acción más allá de la motivación vinculada al escenario, le confiere mayor apertura interpretativa y cierto despegue de búsqueda permanente de desarrollo.

La relación inter-categorial aparece representada con una asociación de las dimensiones resultados-procesos por pares de macrocategorías vinculadas (figura 3). La construcción de la identidad ciudadana está unida a los distintos escenarios por los que transita la persona en la siguiente relación: situada en los distintos ecosistemas, discursiva y dialéctica por el contraste en el ejercicio de roles en escenarios institucionales y comunitarios. Es decir, se trata de una identidad dinámica como participante en la construcción social de escenarios cualitativamente diversos.
El compromiso resulta vinculado con las vivencias que en su perfil diverso, útil, y formativo permiten consolidar los valores del ciudadano participativo. De la observación personal emerge la viabilidad y el vitalismo de los compromisos que asume. En un segundo momento, nuestros informantes experimentan, a través de las vivencias, la sensibilidad hacia los demás y la responsabilidad para con el otro; bajo el referente del futuro por el que evalúan los logros.

La acción está estrechamente relacionada con la socialización en la cultura ciudadana. Los procesos de enculturación se definen, a su vez, por la alfabetización en el liderazgo y el poder de hacer; se desarrollan con la interacción entre las personas a través de comportamientos dialógicos; para concluir, marcando objetivos de cambio y estableciendo proyectos innovadores para alcanzarlos.

Figura 3. El análisis inter-categorial: la vinculación emergente entre categorías

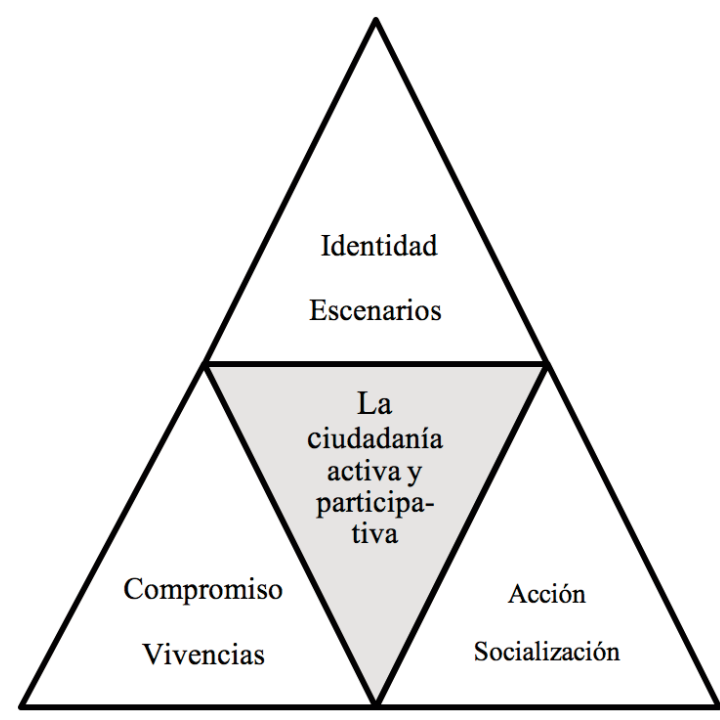

\section{Discusión y conclusiones: pautas para el aprendizaje de la ciudadanía desde la animación sociocultural 5}

El desarrollo de una ciudadanía activa y participativa puede ser relevante en los nuevos retos de la animación sociocultural frente a las prácticas institucionalizadas (Letz \& Pautal, 2012). En uno de los últimos coloquios sobre el tema, Wildemeersch (2012) iniciaba su ponencia "animación y educación" con la misma afirmación que retomaba y enfatizaba Caride (2012, p. 60) en su réplica: “Vivimos en un mundo diferente. Lo mínimo que podemos decir es que vivimos en sociedades complejas". Hecho que obliga a replanteamientos de "las perspectivas y prácticas sociopedagógicas críticas” (Caride, 2012, p. 60) y por tanto del sentido de la animación sociocultural; y en su conjunto de la acción socioedu- 
cativa ante los nuevos escenarios (Morin, Ciurana \& Motta, 2003).

Los resultados obtenidos están en sintonía con otros estudios realizados sobre la formación de la ciudadanía (Holford \& Veen, 2003; Lawy \& Biesta, 2006); en particular, la acción ciudadana como constante en la trayectoria vital, el potencial de la propia acción como formación ciudadana y el protagonismo activo del aprendiz. De la interpretación más amplia del modelo categorial obtenido se desprenden referentes útiles para dinamizar las intervenciones socio-educativas:

- El self contextualizado (desde las macrocategorías identidad y escenarios). Es evidente que en los procesos colectivos de interacción construimos no solo nuestra propia identidad sino la de los demás bajo el bagaje común de una cultura. En este caso, estos procesos tienen su referente educativo en la interdependencia ("connectedness") como promotor del bienestar personal (Townsend \& McWriter, 2005). Los interlocutores ejemplifican estos procesos. Construyen su identidad desde la interacción reflexionada en sus contextos. En cada contexto, ejercen roles específicos de cuya sinergia emerge la construcción de su propia identidad. Se distancian de la identidad instantánea que refiere Ortega (2004), no están descolocados de su trayectoria vital, ni bloqueados o enajenados de sí mismos o de los demás.

La animación sociocultural puede dinamizar este tipo de aprendizaje diversificando los distintos contextos educativos, enriqueciendo el ejercicio de roles diversos, y promoviendo la reflexión sobre sus acciones. Al apreciar la vivencia de múltiples roles, la persona construye una identidad que da cabida a los demás, con las consecuencias críticas en una participación ciudadana no tanto organizada por las instituciones, sino tejida entre todos. Es una identidad marcada por la ética de las responsabilidades (Campillo, 2003). No se trata tan solo de organizar una animación planificada por programas institucionalizados; hay que potenciar la implicación en los contextos reales y próximos a la persona para dar sentido a su compromiso existencial.

- La reinterpretación del pragmatismo (desde las macrocategorías acción y socialización). Los interlocutores introducen un sentido reinterpretado, aparentemente contradictorio pero clarificado a lo largo de las trayectorias de vida contadas: la utilidad empieza por uno mismo, continúa en las personas próximas y avanza hacia el conjunto. Estos protagonistas parecen motivados por metas múltiples (Lunenburg, 2011), en particular las de aprendizaje/dominio y las sociales: las primeras orientadas por el valor de la tarea y la satisfacción que les produce su realización; las segundas, tanto asertivas para realizar su autodeterminación, como integradoras desde su sentido de justicia y equidad. El compromiso ciudadano es la razón que justifica la necesidad de tener poder para hacer cosas útiles porque mejoran la realidad, a la vez que abren nuevas posibilidades de hacer ("agency", "empowerment" y "advocacy").

La animación sociocultural puede promover claves educativas vinculadas a este sentido de pragmatismo. Se trata de fundamentar la formación de la ciudadanía desde el estilo de vida de un ciudadano competente. Estos interlocutores reinterpretan el sentido ciudadano como un estilo de vida que les lleva a actuar con y para los demás; el beneficio personal se transforma en una manera de vivir competente. Es el sentido weberiano de competencia como fenómeno relacional considerando que, como recoge Attewell (2009, p. 40), "la mayoría de las competencias y la honra percibidas son para tareas que no están siendo bien realizadas, donde la incertidumbre de los resultados es alta debido a la escasez de rutinas seguras y conocimiento transmisible".

- La dialéctica del descontrol (desde las macrocategorías acción y socialización). Desde que Theodor Adorno enunciara los principios de la "dialéctica negativa", la superación clásica de la contradicción (tesis-antítesis-síntesis) se sustituye en el razonamiento por el énfasis en la misma, con el consiguiente conflicto en el pensamiento y la emergencia de los dilemas en el sentido de la acción. Este aprendizaje, en el caso de los informantes, es constante y con un matiz de interés: se trata de un aprendizaje apegado a las experiencias que han tenido en situaciones comprometidas, deficitarias y provocadoras de la acción-reflexión. Es el “aprendizaje situado" (Lave \& Wenger, 1991) que vincula el aprender con escenarios lo más reales posible. Si las realidades -como la vida- son conflictivas, imperfectas e imprevisibles, por definición y experiencia vital, el aprendizaje se ha de producir en esas situaciones.

La animación sociocultural puede recuperar aquí la tradición de las situaciones de aprendizaje provocadoras en sí mismas por evidenciar las deficiencias del statu quo. Aprender en estos escenarios es clave. Cuando la realidad es difícilmente controlable, porque así lo demuestra el día a día, aprender en el control aparente resta posibilidades. Es, desde el referente de la complejidad (Nicolescu, 1998), la dialéctica del descontrol que, estimulando el conflicto, puede aplicar la lógica de la incertidumbre y la apertura de la acción socioeducativa a través de la animación sociocultural.

- El principio de la diversificación (desde las macrocategorías vivencias y compromiso). La apuesta por una acción socioeducativa reorientada bajo los referentes emergentes de las historias de vida analizadas debe propiciar:

- Hacer cosas, desde la iniciativa de los protagonistas. La persona en formación para una ciuda- 
danía activa y participativa tiene que experimentar el poder de hacer cosas y no sólo el hacer las cosas que se le permiten o incluso se le sugieren bajo el manto de la múltiple oferta de actividades formativas y educativas.

- Hacer cosas comprometidas, porque tengan finalidad y objetivos claros. De manera más específica, que reviertan sobre compromisos concretos, en procesos de ida y vuelta consigo mismo, con los otros y con el mundo. Es la implicación responsable que Gallardo (2009) marca como último escalón de la solidaridad, después del sentimiento compasivo, la actitud racional del reconocimiento y el valor de la universalización. Para ello, es necesario animar a:

- Hacer cosas en escenarios conflictivos, que reflejen la realidad; pero que a la vez le ofrezcan a la persona en formación ciudadana la posibilidad de hacer posible el cambio comunitario sobre el estado institucional de las cosas. De manera que la persona construya, deconstruya y reconstruya su identidad desde unas vivencias comprometidas con la acción por el cambio (Sabirón \& Arraiz, 2012); vivencias sobre las que la persona reflexionará y construirá su narrativa.

Por último, cabe señalar el potencial y algunas de las limitaciones del estudio:

- El potencial del método narrativo: Pocas técnicas y estrategias de afrontamiento profesional son desechables, desde el pluralismo autocrítico referido por Caride (2003). En este caso, las vivencias a lo largo del proceso de investigación han evidenciado un aprendizaje singular: escuchando, en diálogo con las personas que iban narrando su historia de vida, se aprendía el valor de la persona frente a cualquier imperativo sistémico. En este sentido, la animación sociocultural puede enriquecerse con el método narrativo que, más allá de la ejemplaridad histórico-antropológica, implica a las personas en un diálogo formativo. El auge de este método, evidenciado en su creciente aplicación tanto a la investigación como a la formación le ofrece al profesional de la animación sociocultural recursos viables a utilizar en su práctica (Abril, 2013). Resulta ilustrativa la referencia de Delory- Momberger (2014, p. 703) al proceso de comprensión de una historia de vida: "No puedo (re)construir el mundo de experiencia del relato que recibo más que relacionándolo con mi propia biografía de experiencia y haciéndolo entrar en ella, comprendiéndolo en relaciones de resonancia y de inteligibilidad con mis propias experiencias".

- La optimización del método narrativo no es óbice para señalar las limitaciones del estudio relacionadas con la profundización en cada una de las categorías construidas con los informantes: Se aporta un modelo interpretativo comprehensivo sobre la formación del ciudadano activo; pero a la vez que se refleja la complejidad fenoménico-educativa, se limitan las matizaciones en cada categoría. Tal debilidad orienta hacia la necesidad de continuar con investigaciones análogas, en las que otras historias de vida aporten el desarrollo intrínseco de cada categoría; a la vez se sugiere la pertinencia de estudios que desde diseños de investigación-acción confirmen la utilidad de las categorías resultantes en esta investigación.

\section{Referencias bibliográficas}

Abril, D. (2013). Educación y ciudadanía activa. Reflexiones y propuestas a partir de historias de vida [Education and active citizenship. Reflections and proposals from life stories] (Doctoral thesis). Retrieved from http://espacio.uned.es/fez/view.php?pid=tesisuned:Educacion-Dabril

Attewell, P. (2009). ¿Qué es una competencia? [What is skill?] Pedagogía Social. Revista interuniversitaria, 16, 21-44. doi: $10.7179 /$ PSRI.

Becker, H.S. (1974). Historias de vida en sociología [Life stories in sociology]. In J. Balán (Ed.), Las historias de vida en ciencias sociales (pp. 27-41). Buenos Aires: Ediciones Nueva Visión.

Bertaux, D. (1989). Los relatos de vida en el análisis social [The life stories in the social analysis]. Historia y fuente oral, 1, 8796. Retrieved from http://www.jstor.org/stable/i27753224

Bertaux, D. (1999). El enfoque biográfico: su validez metodológica, sus potencialidades [Biographical approach: its methodological validity, its potentialities]. Proposiciones, 29, 1-23. Retrieved from http://preval.org/files/14BERTAU.pdf

Campillo, M. (2003). Ante una nueva sociedad, una nueva ciudadanía, una nueva respuesta [Facing up a new society, a new citizenship, a new response]. Pedagogía Social. Revista interuniversitaria, 10, 223-232. doi: 10.7179/PSRI.

Caride, J.A. (2003). El pluralismo teórico como argumento epistemológico en el quehacer pedagógico-social [Theoretical pluralism as an epistemological argument in the socio-pedagogical work]. Pedagogía Social. Revista interuniversitaria, 10, 123-159. doi: 10.7179/PSRI.

Caride, J.A. (2012). De la educación a la animación en sociedades complejas: o la reivindicación de un quehacer pedagógico y sociocultural crítico [From education to animation in complex societies or the vindication of a critical pedagogical and socio-cultural work]. In F. Curto (Ed.), V Coloquio Internacional de Animación Sociocultural: cultura, educación y ciudadanía (pp. 59-69). Zaragoza: Diputación Provincial de Zaragoza Área de Cultura y Patrimonio. 
Delory-Momberger, C. (2014). Experiencia y formación [Experience and training]. Revista Mexicana de Investigación Educativa, 19(62), 695-710.

Ferrarotti, F. (2009). El conocimiento socioantropológico como conocimiento participado y verdad intersubjetiva [Socioanthropological knowledge as participated and intersubjective knowledge]. Historia, antropología y fuentes orales, 41, 107-122.

Gallardo, P. (2009). Educación ciudadana y convivencia democrática [Citizenship education and democratic coexistence]. Pedagogía Social. Revista interuniversitaria, 16, 119-133. doi: 10.7179/PSRI.

Gil-Jaurena, I., Aguado, T., Mata, P., \& Ballesteros, B. (2011, Octubre). Investigación sobre aprendizaje en ciudadanía activa [Research on active citizenship learning]. Paper presented at the $V$ Coloquio internacional de animación sociocultural: cultural, educación y ciudadanía, Zaragoza. Retrieved from http://www.unizar.es/colinanimacion-IEPSA/?page_id=1583

Glaser, B., \& Strauss, A. (2002). The Discovery of Grounded Theory and Appllying Grounded Theory. In N.K. Denzin \& Y.S. Lincoln (Eds.), The American Tradition in Qualitative Research, (vol. II, pp. 229-243). London: Sage Pu.

Holford, J., \& van der Veen, R. (2003). Lifelong learning, governance and active citizenship in Europe. Final report of the ETGACE Research Project: Analysis of Adult Learning \& Design of Formal, Non-Formal \& Informal Educational Intervention Strategies. Supported by the European Commission (Directorate General for Research) Framework Programme, 5. Retrieved from ftp://ftp.cordis.europa.eu/pub/citizens/docs/hpse-ct-1999-00012etgace_eur21533final.pdf

Huberman, A.M., \& Miles, M.B. (1991). Analyse des données qualitatives [Handling qualitative data]. Bruxelles: De Boeck.

Josso, M.C. (2002). Experiências de vida e formaçao [Life and training experiences]. Lisboa: Educa.

Lave, J., \& Wenger, E. (1991). Situated Learning. New York: Cambridge University Press.

Lawy, R., \& Biesta, G. (2006). Citizenship-as-Practice: The Educational Implications of an Inclusive and Relational Understanding of Citizenship. British Journal of Educational Studies, 54(1), 34-50. doi: 10.111/j.1467-8527.2006.00335.x

Letz, C., \& Pautal, E. (2012). Peut-on apprendre la citoyenneté? Trois modèles d'éducation politique et sociale pour les élites [Can you learn citizenship? Three models of social and political education for the elite]. La Recherche en Éducation, 8, 44-53. Retrieved from http://www.la-recherche-en-education.org/index.php/afirse/article/view/117/79

Lunenburg, F. (2011). Goal-Setting Theory of Motivation. International Journal of Management, Business and Administration, 15(1), 1-6. Retrieved from http://www.nationalforum.com/

Marie-Claude, B. (2014). La présentation de soi: cadre pour aborder l'analyse de récits de vie [The presentation of the self: chart to address the analysis of life stories]. Interrogations?, 17. Retrieved from http://www.revue-interrogations.org/Lapresentation-de-soi-cadre-pour

Mata, P., \& Gil-Jaurena, I. (Eds.) (en prensa). Aprendizaje de la ciudadanía activa: discursos, experiencias y estrategias educativas [Active citizenship learning: speeches, experiences, and educational strategies]. Madrid: UNED.

Morin, F. (1980). Pratiques anthropologiques et histoire de vie [Anthropological practices and life history]. Cahiers internationaux de sociologie, LXIX, 313-339. Retrieved from http://classiques.uqac.ca/contemporains/morin_francoise/ pratiques_anthropo_hist_de_vie/pratiques_anthropo_texte.html.

Morin, E., Ciurana, E., \& Motta, R. (2003). Educar en la era planetaria [Educating on the planetary era]. Barcelona: Gedisa.

Nicolescu, B. (1998). Le tiers inclus. De la physique quantique à I'ontologie [The included middle. From the Quantum Physics to Ontology]. Bulletin interactive du CIRET, 13. Retrieved from http://ciret-transdisciplinarity.org/bulletin/b13c11.php

Ortega, P. (2004). Educar para la participación ciudadana [Educating for citizenship participation]. Pedagogía Social. Revista interuniversitaria, 11, 215-236. doi: 10.7179/PSRI.

Peavy, V. (1998). Sociodynamic counseling: a constructivist perspective. Victoria Canadá: Trafford.

Peneff, J. (1990). La méthode biographique [The biographical method]. París: Armand Colin.

Pineau, G. (2006). Les histoires de vie en formation: genèse d'un courant de recherche-action-formation existentielle [Life stories training: genesis of an action-research-existential training trend], Educaçao e Pesquisa, 32(2), 329-343.

Richards, L., (2010). Handling qualitative data. London: Sage Pu.

Sabirón, F. (2006). Métodos de investigación etnográfica en ciencias sociales [Methods of ethnographic research in Social Sciences]. Zaragoza: Mira editores.

Sabirón, F., \& Arraiz, A. (2012). La complexité dans la pratique éducative: naviguer entre dilemmes [The complexity in the educative practice: to navigate between dilemmas]. In Y. Lenoir \& F. Tupin (Eds.), Les pratiques enseignantes entre instruire et socialiser (pp. 255-288). Québec: Les Presses de l'Université Laval.

Stake, R.E. (1998). Investigación con estudio de casos [Case studies research]. Madrid: Morata.

Townsend, K., \& McWriter, B. (2005). Connectedness: A Review of the Literature With Implications for Counseling, Assessment, and Research. Journal of Counseling \& Development, 83(2), 191-201.

Úcar, X. (2002). Medio siglo de animación sociocultural en España: balance y perspectivas [Half a century of socio-cultural animation in Spain: balance and perspectives]. Revista lberoamericana de Educación, 2, 1-22. Retrieved from http://www.rieoei.org/deloslectores/301Ucar.pdf.

Villers, G. (2011). L'approche autobiographique: regards anthropologique et épistémologique, et orientations méthodologiques [Autobiographical approach: epistemological and anthropological perspectives and methodological guidelines]. Recherches sociologiques et anthropologiques, 42(7), 25-44. 
Wildemeersch, D. (2012). Animación y educación en sociedades complejas: un recorrido por las perspectivas y prácticas sociopedagógicas críticas [Animation and education in complex societies: an overview of perspectives and critical sociopedagogical practices]. In F. Curto (Ed.), V Coloquio Internacional de Animación Sociocultural: cultura, educación y ciudadanía (pp. 35-57). Zaragoza: Diputación Provincial de Zaragoza Área de Cultura y Patrimonio.

\section{Notas}

1 “Aprendizaje de la ciudadanía activa. Discursos, experiencias y estrategias educativas" (Plan Nacional de I+D+i, Subdirección General de Proyectos de Investigación del Ministerio de Educación, referencia EDU200909195. El grupo INTER de Investigación en Educación Intercultural coordinó el proyecto, en cuya web se encuentra disponible la documentación y la fundamentación teórica del estudio (http://www.uned.es/grupointer/aprendiz_ciudadania_activa.html).

2 Es reseñable el proyecto coordinado por Christian Leray "biografía y ciudadanía" de la "red de escuelas de ciudadanía". La presentación del proyecto está disponible en http://www.recit.net/?Atelier-thematiqueRECit-et\&lang=fr y en http://www.asihvif.com/1/upload/biographiecitoyenneteatelier.pdf.

3 Para preservar la privacidad, los nombres no se corresponden con la realidad. Fue decisiva la disponibilidad de Marta, María y Miguel para dedicarnos su tiempo, pero sobre todo, para compartir su vida. Nuestro agradecimiento.

4 En el sistema convencional, se satura la categoría por el número de registros. Sin embargo, en las historias de vida, el criterio que hemos aplicado es la intersubjetividad; es decir, la categoría se valida por el significado triangulado entre la persona historiada, el texto y el investigador (Sabirón, 2006).

5 La animación sociocultural planteada como una metodología transversal de intervención, según la tendencia preferente consignada por Úcar (2002).

\section{CÓMO CITAR ESTE ARTÍCULO}

Arraiz, A., Azpillaga, V., \& Sabirón, F. (2016). El aprendizaje de la ciudadanía a lo largo de la vida: Un estudio narrativo desde historias de vida. Pedagogía Social. Revista Interuniversitaria, 28 189-201. DOI:10.7179/PSRI_2016.28.14

\section{DIRECCIÓN DE LOS AUTORES}

Ana Arraiz Pérez: Universidad de Zaragoza. E-mail: aarraiz@unizar.es

Verónica Azpillaga Larrea. Universidad del Pais Vasco / Euskal Herriko Unibertsitatea.

E-Mail: veronica.azpillaga@ehu.es

Fernando Sabirón Sierra. Universidad de Zaragoza. E-mail: fsabiron@unizar.es

\section{PERFIL ACADÉMICO}

Ana Arraiz Pérez. Profesora Titular de Universidad. Investigadora del Grupo de Investigación Aplicada en Etnografía de la Educación. Líneas de investigación cualitativa en orientación, evaluación y métodos de investigación en los ámbitos de la educación formal, no-formal e informal.

Verónica Azpillaga Larrea. Profesora Titular de Universidad. Líneas de investigación cualitativa en orientación, evaluación y métodos de investigación en los ámbitos de la educación formal, no-formal e informal.

Fernando Sabirón Sierra. Profesor Titular de Universidad. Director del Dpto. de Ciencias de la Educación de la Universidad de Zaragoza. Investigador principal del Grupo de Investigación Aplicada en Etnografía de la Educación. Líneas de investigación cualitativa en orientación, evaluación y métodos de investigación en los ámbitos de la educación formal, no-formal e informal. 
